\title{
Physical properties of Southern infrared dark clouds ${ }^{\star}, \star \star$
}

\author{
T. Vasyunina ${ }^{1, \star \star \star}$, H. Linz ${ }^{1}$, Th. Henning ${ }^{1}$, B. Stecklum ${ }^{2}$, S. Klose ${ }^{2}$, and L.-Å. Nyman ${ }^{3}$ \\ 1 Max Planck Institute for Astronomy (MPIA), Königstuhl 17, 69117 Heidelberg, Germany \\ e-mail: [vasyunina, linz, henning] @mpia.de \\ 2 Thüringer Landessternwarte Tautenburg, Sternwarte 5, 07778 Tautenburg, Germany \\ e-mail: [stecklum;klose]@tls-tautenburg.de \\ 3 ESO, Santiago 19, 19001 Casilla, Chile \\ e-mail: lnyman@eso.org
}

Received 24 October 2008 / Accepted 6 February 2009

\section{ABSTRACT}

\begin{abstract}
Context. What are the mechanisms by which massive stars form? What are the initial conditions for these processes? It is commonly assumed that cold and dense Infrared Dark Clouds (IRDCs) represent the birth-sites of massive stars. Therefore, these clouds have been receiving an increasing amount of attention, and their analysis offers the opportunity to tackle the afore mentioned questions. Aims. To enlarge the sample of well-characterised IRDCs in the southern hemisphere, where ALMA will play a major role in the near future, we have developed a program to study the gas and dust of southern infrared dark clouds. The present paper attempts to characterize the continuum properties of this sample of IRDCs.

Methods. We cross-correlated $1.2 \mathrm{~mm}$ continuum data from SIMBA bolometer array mounted on SEST telescope with Spitzer/GLIMPSE images to establish the connection between emission sources at millimeter wavelengths and the IRDCs that we observe at $8 \mu \mathrm{m}$ in absorption against the bright PAH background. Analysing the dust emission and extinction enables us to determine the masses and column densities, which are important quantities in characterizing the initial conditions of massive star formation. We also evaluated the limitations of the emission and extinction methods.

Results. The morphology of the $1.2 \mathrm{~mm}$ continuum emission is in all cases in close agreement with the mid-infrared extinction. The total masses of the IRDCs were found to range from 150 to $1150 M_{\odot}$ (emission data) and from 300 to $1750 M_{\odot}$ (extinction data). We derived peak column densities of between 0.9 and $4.6 \times 10^{22} \mathrm{~cm}^{-2}$ (emission data) and 2.1 and $5.4 \times 10^{22} \mathrm{~cm}^{-2}$ (extinction data). We demonstrate that the extinction method is unreliable at very high extinction values (and column densities) beyond $A_{\mathrm{V}}$ values of roughly 75 mag according to the Weingartner \& Draine (2001) extinction relation $R_{\mathrm{V}}=5.5$ model B (around 200 mag when following the common Mathis (1990, ApJ, 548, 296) extinction calibration). By taking the spatial resolution effects into account and restoring the column densities derived from the dust emission to a linear resolution of $0.01 \mathrm{pc}$, peak column densities of $3-19 \times 10^{23} \mathrm{~cm}^{-2}$ are obtained, which are much higher than typical values for low-mass cores.

Conclusions. Taking into account the spatial resolution effects, the derived column densities are beyond the column density threshold of $3.0 \times 10^{23} \mathrm{~cm}^{-2}$ required by theoretical considerations for massive star formation. We conclude that the values of column densities derived for the selected IRDC sample imply that these objects are excellent candidates for objects in the earliest stages of massive star formation.
\end{abstract}

Key words. ISM: dust, extinction - ISM: clouds - infrared: ISM - radio continuum: ISM - stars: formation

\section{Introduction}

One of the key challenges in stellar astrophysics is to understand the formation and earliest evolution of high-mass stars. These objects play a major role in shaping the interstellar medium due to their strong UV radiation fields and stellar winds, and enrich their environment with heavy elements as they explode as supernovae. Despite their importance, the mechanism by which such massive stars form and, especially, the initial conditions

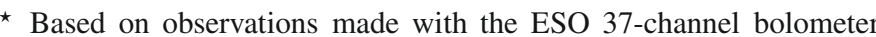
array SIMBA at the SEST telescope on La Silla, under programme ID 71.C-0633.

$\star \star$ FITS files of the SEST maps of Figs. 1 and 2 are only available in electronic form at the CDS via anonymous ftp to cdsarc.u-strasbg.fr $(130.79 .128 .5)$ or via http://cdsweb.u-strasbg.fr/cgi-bin/qcat?J/A+A/499/149

$\star \star \star$ Member of the International Max Planck Research School (IMPRS) Heidelberg.
}

of their birthplaces are poorly understood (Beuther et al. 2007; Zinnecker \& Yorke 2007).

One possible means of studying the initial conditions of massive stars is to analyse the so-called Infrared Dark Clouds (IRDCs). These were first identified by the Infrared Space Observatory (ISO; Perault et al. 1996) and Midcourse Space Experiment (MSX; Egan et al. 1998) as dark extended features against the bright Galactic PAH background at mid-IR (MIR) wavelengths. The first studies (Egan et al. 1998; Carey et al. $1998,2000)$ showed that IRDCs are dense $\left(>10^{5} \mathrm{~cm}^{-3}\right)$, cold $(<25 \mathrm{~K})$, and can attain high column densities $\left(\gtrsim 10^{23} \mathrm{~cm}^{-2}\right)$. All of these properties imply that IRDCs are excellent candidates for hosting the very early stages of massive star formation.

Studies of Infrared Dark Clouds have also been completed at millimeter and submillimeter wavelengths. Simon et al. (2006a) presented a catalog of almost 11000 IRDCs in the first and fourth quadrants of the Galactic plane. Using ${ }^{13} \mathrm{CO} J=1-0$ molecular line emission, the kinematic distances to 313 clouds in this catalog were established (Simon et al. 2006b). This 
allowed to estimate the sizes, masses, and Galactic distribution for this large sample. The study showed that IRDCs have sizes of $\sim 5 \mathrm{pc}$ and LTE masses of $\sim 5 \times 10^{3} M_{\odot}$, which are both comparable to the typical values for cluster-forming molecular clumps. The Galactic distribution of IRDCs follows the general distribution of molecular gas. A concentration of the clouds is associated with the so-called $5 \mathrm{kpc}$ molecular ring, the Galaxy's most massive and active star-forming complex. Ammonia observations of some well-known IRDCs with the Effelsberg $100 \mathrm{~m}$ telescope (Pillai et al. 2006a) allowed to estimate additional chemical and physical properties, such as average gas temperature (between 10 and $20 \mathrm{~K}$ ), velocity fields (significant velocity gradient between the cores, linewidths of $0.9-1.5 \mathrm{~km} \mathrm{~s}^{-1}$ ), and the chemical state. According to this study, $\mathrm{NH}_{3}$ in IRDCs is overabundant by a factor of 5-10 relative to Taurus or Perseus local dark clouds, while $\mathrm{H}_{2} \mathrm{CO}$ is underabundant by a factor of $\sim 50$. Hence, the chemistry governing IRDCs might be complex and could differ from that in other parts of the molecular ISM.

Although significant progress has been made observationally, the number of IRDCs with well characterised properties remains small, especially those observable from the southern hemisphere. To enlarge the sample of well-investigated IRDCs, we selected 12 clouds in the southern hemisphere and started a program to measure the gas and dust properties of these objects.

In Sect. 2, we describe our source selection and $1.2 \mathrm{~mm}$ continuum observations with the SIMBA/SEST telescope. In Sect. 3, we discuss the data reduction and the details concerning the calculation of dust masses and column densities. We also present a comparison between the MIR and millimeter techniques. In Sect. 4, we compare our results with previous results for high- and low-mass star-forming region and theoretical models.

\section{Observations}

The IRDCs for our study were selected in the pre-Spitzer era, by visual examination of the MIR images delivered by the MSX satellite. The MSX A band (6.8-10.8 $\mu \mathrm{m})$ was the most sensitive of all the MSX bands and exhibits the highest level of diffuse background emission (due to PAH emission at 7.7 and $8.7 \mu \mathrm{m}$ ), which also produces the highest contrast between bright background and dark IRDCs. We selected a sample of southern IRDCs from the A band images by searching for high contrast and sizes sufficient to fill the main beam of the SEST telescope at $1.2 \mathrm{~mm}$.

The $1.2 \mathrm{~mm}$ continuum observations were carried out with the 37-channel bolometer array SIMBA (Nyman et al. 2001) at the SEST on La Silla, Chile between July 16-18, 2003. SIMBA is a hexagonal array in which the half-power beamwidth (HPBW) of a single element is about 24" and the separation between elements on the sky is $44^{\prime \prime}$. The observations were completed using a fast-mapping technique without a wobbling secondary (Weferling et al. 2002).

Maps of Uranus were taken to check the flux calibration of the resulting data. To correct for the atmospheric opacity, skydips were performed every 2-3 h. Despite the occurrence of some thin clouds, the observing conditions were good, which is reflected in the zenith opacity values of $0.16-0.18$. The pointing was checked roughly every two hours and was found to be superior to 6 ". The combination of typically three maps of sizes $560^{\prime \prime} \times 900^{\prime \prime}$ resulted in a residual noise of about $22-28 \mathrm{mJy} / \mathrm{beam}(\mathrm{rms})$ at the center of the mapped region.

\section{Data reduction and analysis}

We use both $8 \mu \mathrm{m}$ IRAC data from the Spitzer Galactic Legacy Infrared Mid-Plane Survey Extraordinaire (GLIMPSE, Benjamin et al. 2003) and our $1.2 \mathrm{~mm}$ data from the SIMBA bolometer at the SEST telescope to investigate the physical properties of the extinction and emission material.

To derive the masses of the IRDCs, in both cases we need to determine the distances to these clouds. To estimate the (kinematic) distances to our IRDCs, we use the $v_{\mathrm{LSR}}$ velocities derived from recent molecular line observations ${ }^{1}$ acquired with the Australian MOPRA telescope, which we present in a forthcoming paper. The velocities were transferred to kinematic distances by adopting the improved parameters for the Galactic rotation curve (Levine et al. 2008) for the fourth and first Galactic quadrant. The closest kinematic distance was always assumed. The corresponding distances used in the mass estimations are reported in Table 3 . We note that these rotation curves provide average properties. The true distribution of material might be more structured, especially in the fourth quadrant, which is indicated in the HI absorption measurements shown in Levine et al. (2008). Furthermore, for objects in the Galactic longitude interval $\left[305^{\circ}, 310.5^{\circ}\right]$, several velocity systems can occur due to the projection of at least two Galactic arms. The measured velocities, however, place all our IRDCs in that longitude range within the Centaurus arm (3.5-5.5 kpc), in agreement with Saito et al. (2001).

\subsection{Millimeter data}

The $1.2 \mathrm{~mm}$ data for the IRDC regions from SIMBA at the SEST telescope were reduced using the MOPSI package (developed by Zylka, IRAM). All maps were reduced by applying the atmospheric opacity corrections, fitting and subtracting a baseline, and removing the correlated sky noise. Thereby, we followed a three-stage approach as suggested in the SIMBA manual. After a first iteration using all data for the sky-noise removal, the map regions showing source emission are neglected for sky-noise removal in the second iteration. From this second interim map, a source model is derived, which is included in the third iteration. The resulting maps were flux-calibrated using the conversion factor obtained from observations of Uranus. For our July 2003 observations, this factor was around $60 \mathrm{mJy} /$ beam per count.

For estimating cloud masses and column densities we used the following expressions:

$M_{\mathrm{tot}}=\frac{F_{\mathrm{int}} D^{2} R}{B_{v}(T) \varkappa_{v}}$

$N_{\mathrm{H}_{2}}=\frac{F_{\text {peak }} R}{\Omega B_{v}(T) \varkappa_{v} m_{\mathrm{H}_{2}}}$.

The measured source peak flux density is given by $F_{\text {peak }}, F_{\text {int }}$ denotes the integrated flux density of the complete source, $\Omega$ is the beam solid angle in steradians, $m_{\mathrm{H}_{2}}$ is the mass of one hydrogen molecule, $D$ is the distance to the IRDC, $R$ is the gasto-dust ratio, $\varkappa_{v}$ is the dust opacity per gram of dust, and $B_{v}(T)$ is the Planck function at the dust temperature $T$. We adopt a gasto-dust mass ratio of 100 , and $\varkappa_{v}$ equal to $1.0 \mathrm{~cm}^{2} \mathrm{~g}^{-1}$, a value appropriate for cold dense cores (Ossenkopf \& Henning 1994).

At the present stage, where measured temperatures are unavailable for our sources, we assume a temperature of $20 \mathrm{~K}$, which is a reasonable choice considering investigations toward

1 The $\mathrm{HCO}^{+}(1-0)$ line velocities have been employed for this purpose. 
other IRDCs (Carey et al. 1998; Pillai et al. 2006b). The derived mass depends on the (assumed) temperature, on the distance to the cloud, and on the grain model. Masses are underestimated if the temperature is lower than the assumed value of $20 \mathrm{~K}$. For example, at $15 \mathrm{~K}$ the masses will be higher by a factor of 1.5 . In case of a higher temperature in the cloud, e.g., for $30 \mathrm{~K}$, our results must be divided by a factor of 1.7 . We further note the quadratic dependence of the derived masses on the distance to the clouds. Hence, the masses will be a factor of 1.2-1.8 higher, if the distance is $500 \mathrm{pc}$ more, and lower by the same factor if it is $500 \mathrm{pc}$ less than indicated by the average Galactic rotation curve (see above). The derived masses are inverse proportional to the assumed value of the opacity $\varkappa_{v}$, which has an uncertainty of at least a factor 2 . The column density has no direct dependence on the distance to the cloud, but the temperature dependence is the same as for the masses.

\subsection{GLIMPSE $8 \mu \mathrm{m}$ data}

The original selection of the IRDCs was completed on the basis of MSX images. In the meantime, the Spitzer satellite has since succeeded MSX and provided a far higher spatial resolution and sensitivity. GLIMPSE images for our regions with a pixel size of 0.' 6 were retrieved from the NASA/IPAC Infrared Science Archive (IRSA) and remosaicked to cover the final field of our $1.2 \mathrm{~mm}$ maps of typically $15^{\prime} \times 15^{\prime}$ to determine how the IRDCs relate to their closer and further vicinity (see Figs. 1, 2). After bad-pixel removal, a PSF photometry was performed using the STARFINDER program (Diolaiti et al. 2000). This allows us to remove compact foreground objects, and thus maps of extended emission and absorption structures, and finally column density maps could be extracted in a subsequent step.

Dust masses for the IRDCs were computed by assuming that they are in the foreground and attenuate emission from behind. The optical depth $\tau$ is ideally the logarithm of the ratio of two fluxes: (a) the flux from the emission background $I_{\text {back }}$ directly behind the IRDC, and (b) the actually measured remnant flux $I_{\text {IRDC }}$ from the location of the IRDC. Furthermore, superimposed on the IRDC is an emission contribution from foreground material, $I_{\text {fore }}$, which must be subtracted. Since $I_{\text {back }}$ cannot be estimated directly, we need a measurable quantity $I_{0}$ that can be used as a proxy for $I_{\text {back }}$. The optical depth is then given by

$\tau=\ln \left(\frac{I_{0}-I_{\text {fore }}}{I_{\text {IRDC }}-I_{\text {fore }}}\right)$.

Following Peretto et al. (2008), we assume that $I_{\text {fore }}=I_{\mathrm{zl}}$, where $I_{\mathrm{zl}}$ is the zodiacal light, which is calculated systematically for every Spitzer observation and available in the image header. For the quantity $I_{0}$, we used the average emission level from a patch of MIR emission in the close vicinity of the actual cloud. These emission patches, typically around 1 square arcminute in size, were chosen manually to capture the characteristic emission level for the background approximation of the individual clouds and to exclude strong compact emission sources. The mean and standard deviation of the emission levels within these defined regions were computed to be used as $I_{0}$ in Eq. (3). The standard deviation obtained here is propagated in the following steps and used to provide formal errors for the derived masses and column densities, as listed in Table 3.

After optical depth determination, this quantity is converted into column densities and finally to masses by using the following equations:

$N_{\mathrm{H}_{2}}=1.086 \frac{\tau}{\sigma}$
$M=m_{\mathrm{H}_{2}} A N_{\mathrm{H}_{2}}$,

where $N_{\mathrm{H}_{2}}$ is the $\mathrm{H}_{2}$ molecule column density, $m_{\mathrm{H}_{2}}$ is the mass of one hydrogen molecule, $A$ is the area per pixel, and $\sigma$ is the extinction cross-section per hydrogen molecule. According to the adopted dust-extinction model by Weingartner \& Draine (2001, see below), $\sigma=4.62 \times 10^{-23} \mathrm{~cm}^{2}$ for the Spitzer/IRAC band 4 central wavelength of $7.87 \mu \mathrm{m}$.

The derived masses depend critically on the adopted extinction-column density calibration, the distance to the targets, and the method for selecting the relevant extinction regions. As a dust model, we used the parametrisation of Weingartner \& Draine (2001) for their model B with $R_{\mathrm{V}}=5.5$. This particular model was shown to be relevant for massive star-forming regions, e.g., by Indebetouw et al. (2005). It differs from the common dust models (e.g., Draine \& Lee 1984) in that it predicts higher extinction cross-sections particularly for the 4-8 micron wavelength region, a relevant point for the Spitzer extinction maps. We note that these elevated cross-sections are also predicted in connection with ice-coated dust grains and especially if dust coagulation processes are involved (Ossenkopf \& Henning 1994). The chosen dust model finally relates the optical depth (and, equivalently, the extinction magnitude at the used wavelength) to the equivalent column density of hydrogen molecules. The quadratic dependence of the masses on the distance is in the extinction map case the same as for masses derived from the $1.2 \mathrm{~mm}$ emission data.

\subsection{Comparison of techniques}

Using the data from two different regions of the spectrum and two different techniques for estimating IRDC parameters, enables us to compare the results and to analyse advantages and disadvantages of both methods (see Table 1). The mid-IR data have an effective resolution of around $3^{\prime \prime}$, which is much higher than $24^{\prime \prime}$ for our millimeter data. The estimated total masses and peak column densities have no dependence on the temperature. On the other hand, when using $8 \mu \mathrm{m}$ data to calculate the optical depth and then the column density in the cloud, we have to estimate the expected flux from the background indirectly. We assume the background flux to be the difference between the average intensity around the cloud and the foreground intensity, although in reality matter hidden by the infrared dark cloud can be inhomogeneous and of either lower or higher intensity. However, the logarithm in Eq. (3) to a certain degree mitigates uncertainties in the estimation of the intensities necessary for evaluating Eq. (3).

Simon et al. (2006a) used an automated approach where they strongly smoothed the original (MSX) mid-infrared images and basically used these smoothed data to approximate for the quantity $I_{0}$ (cf. Eq. (3)). We tested this approach for one of our clouds but found that unless large smoothing kernels were used, the herewith derived intensity contrasts were systematically smaller than for the method that we employed (Sect. 3.2). For very large smoothing kernels $\left(>10^{\prime}\right)$, it is very difficult to control the process. Continuum emission from strong extended emission sources in the surroundings might be folded into the area of the IRDCs, depending on the individual circumstances. Therefore, we refrain from using this smoothing approach to achieve stronger control on the estimation of the quantity $I_{0}$.

Uncertainties in the foreground level estimation also affect the resulting masses and column densities. At the moment, we take into account only the zodiacal light contribution, which typically accounts for $15 \%-20 \%$ of the intensity toward the IRDCs. 

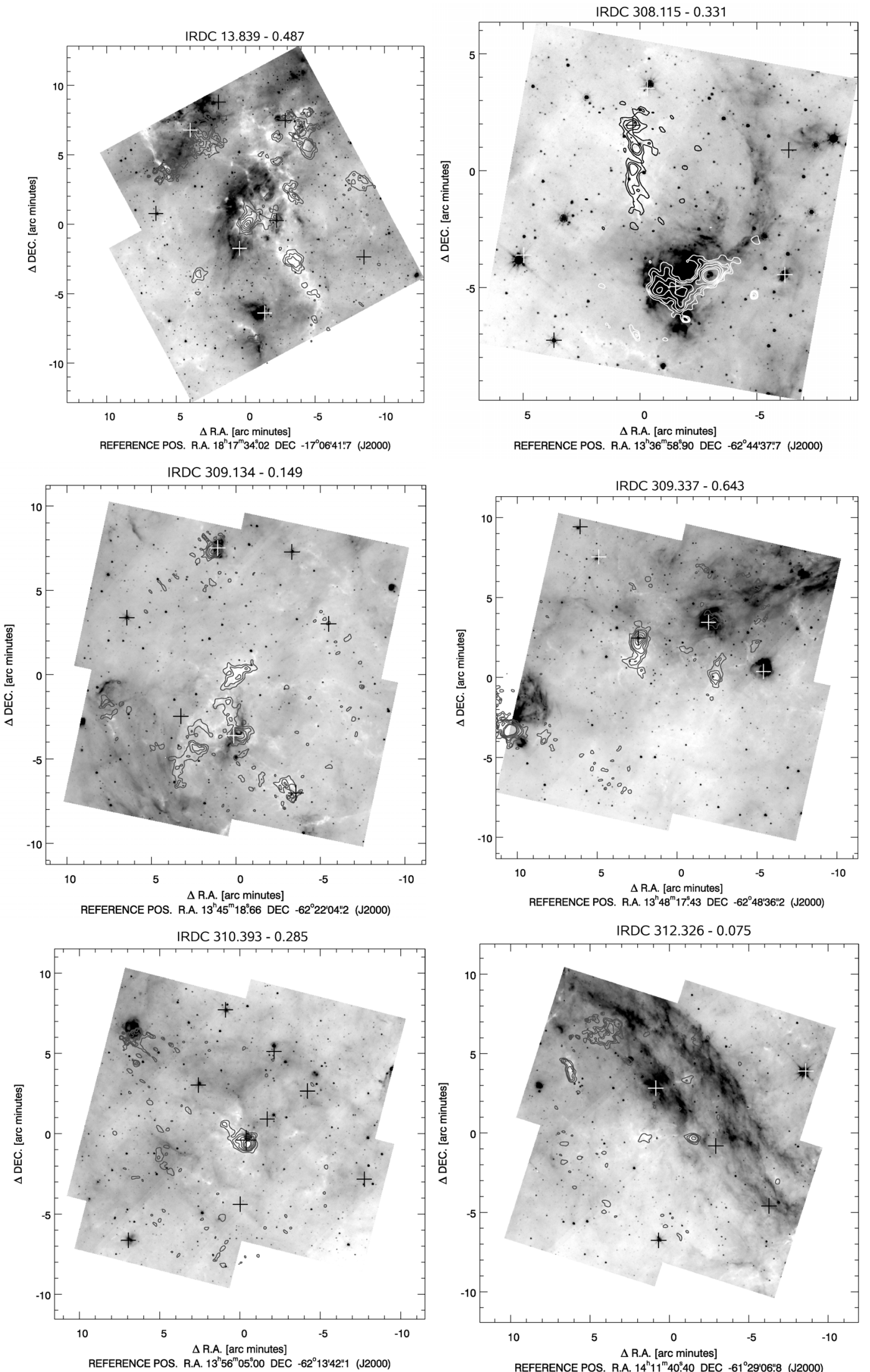

Fig. 1. Inverse grey-scale 8 micron maps overlaid with $1.2 \mathrm{~mm}$ continuum emission. The intensity of a grey-scale image corresponds to the square root of the inverse intensity in mJy. The contours are 60, 108, 156, 240, 360, 480 mJy beam $^{-1}$ in all cases. Crosses denote the position of IRAS point sources. 

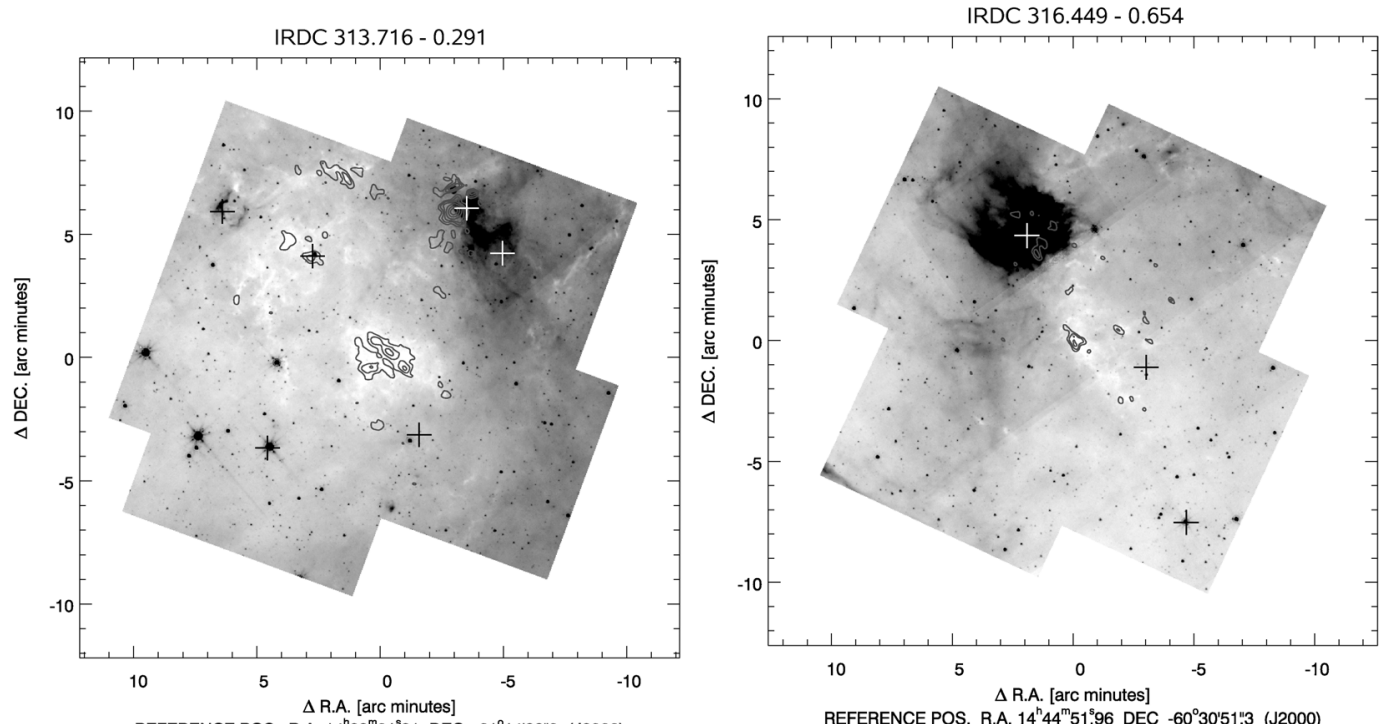

REFERENCE POS. R.A. $14^{n} 23^{\mathrm{m}} 01^{\mathrm{s}} \cdot 31$ DEC $-61^{\circ} 14^{\prime 2} 22^{\prime \prime} 0($ (J2000)
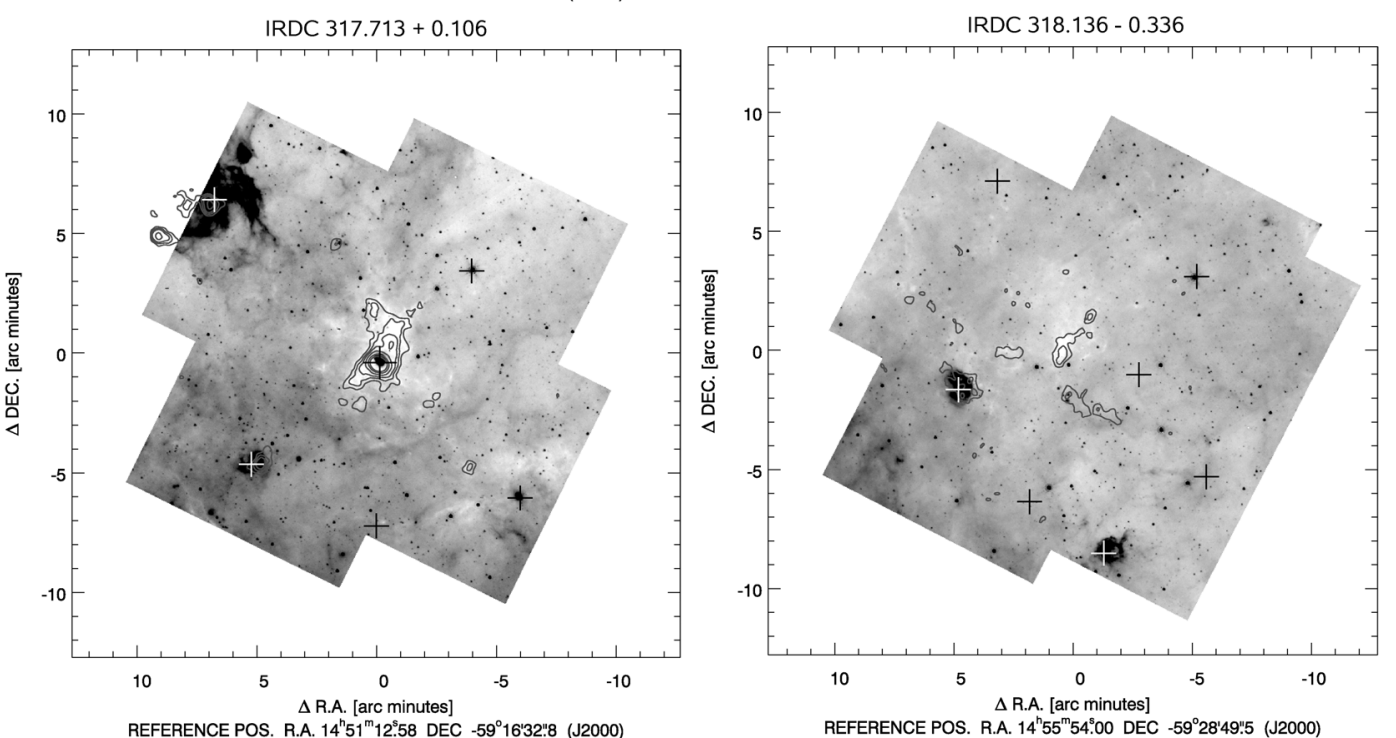

REFERENCE POS. R.A. $14^{\mathrm{n}} 51^{\mathrm{m}} 12^{\mathrm{s}} .58$ DEC $-59^{\circ} 16^{\prime} 32.8$ (J2000)
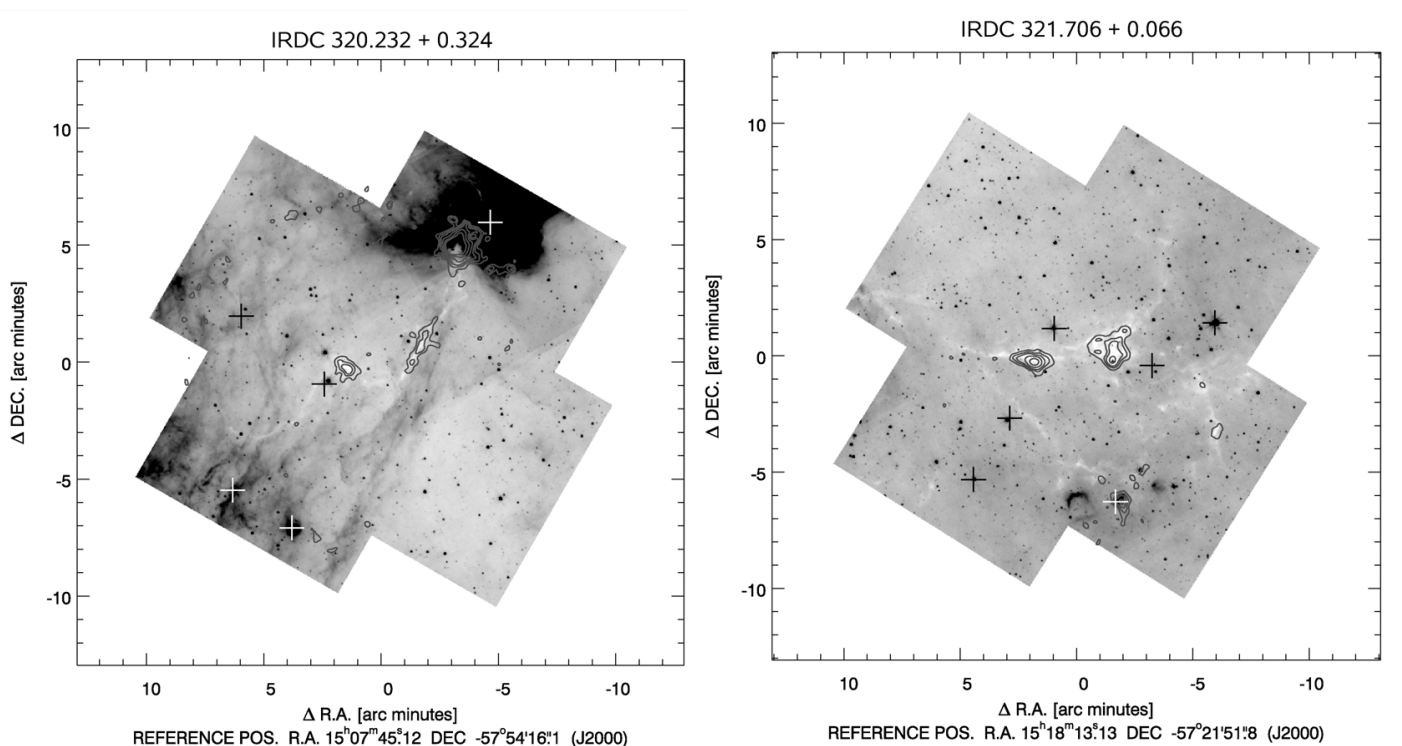

Fig. 2. Inverse grey-scale 8 micron maps overlaid with 1.2. $\mathrm{mm}$ continuum emission. The intensity of a grey-scale image corresponds to the square root of the inverse intensity in mJy. The contours are $60,108,156,240,360,480 \mathrm{mJy}^{-1}$ beam $^{-1}$ in all cases except for IRDC $316.45-0.65$ where it is $84,120,156,240,360,480 \mathrm{mJy}_{\text {beam }}{ }^{-1}$. Crosses denote the position of IRAS point sources. 
Table 1. Comparison of the $1.2 \mathrm{~mm}$ emission and $8 \mu \mathrm{m}$ absorption techniques for deriving masses and column densities.

\begin{tabular}{lcc}
\hline \hline Properties & $1.2 \mathrm{~mm}$ & $8 \mu \mathrm{m}$ \\
\hline Distance dependence ${ }^{a}$ & + & + \\
Resolution & $24^{\prime \prime}$ & $3^{\prime \prime}$ \\
$\begin{array}{l}\text { Sensitive to lower column } \\
\text { density filigree structure }\end{array}$ & - & + \\
Temperature dependence & + & - \\
Background and foreground & not & necessary \\
estimation & necessary & \\
$\begin{array}{l}\text { Sensitive to column } \\
\text { densities } \gg 10^{23} \mathrm{~cm}^{-2}\end{array}$ & + & - \\
\hline for masses & & \\
\hline
\end{tabular}

${ }^{a}$ Only for masses.

Table 2. Comparison of the different observational techniques for IRDC 18223-3.

\begin{tabular}{lcccc}
\hline \hline Data & $\begin{array}{c}\text { Resolution } \\
(\operatorname{arcsec})\end{array}$ & $\begin{array}{c}\text { Column density } \\
\left(10^{22} \mathrm{~cm}^{-2}\right)\end{array}$ & $\begin{array}{c}\text { Optical depth } \\
\text { at } 8 \mu \mathrm{m}\end{array}$ & $\begin{array}{c}\text { Contrast } \\
\left(I_{\mathrm{bg}} / I_{\mathrm{IRDC}}\right)\end{array}$ \\
\hline $8 \mu \mathrm{m} \mathrm{GLIMPSE}$ & 3 & 2.3 & 0.95 & 2.5 \\
$1.2 \mathrm{~mm} \mathrm{IRAM}^{a}$ & 11 & 5.9 & 2.4 & 11 \\
$3.2 \mathrm{~mm} \mathrm{PdBI}^{b}$ & $5^{\prime \prime} 8 \times 22^{\prime \prime} 4$ & 45 & 19 & $1.8 \mathrm{e}+08$ \\
$1.3 \mathrm{~mm} \mathrm{SMA}^{c}$ & $1^{\prime \prime} \cdot 3 \times 1^{\prime \prime} 4$ & 93 & 40 & $1.8 \mathrm{e}+17$ \\
\hline
\end{tabular}

${ }^{a}$ Beuther et al. (2002); ${ }^{b}$ Beuther et al. (2005); ${ }^{c}$ Fallscheer et al. (2009, in preparation).

An extreme (hypothetical) case may be that all remaining flux received from the IRDC to the noise level $I_{\text {noise }}$ of the GLIMPSE images is produced by another foreground emission contribution (e.g., the PDR surface of the cloud, glowing in PAH emission). After removing all this emission down to $I_{\text {noise }}$, the resulting intensity contrast would of course be clearly higher. However, considering typical levels of $I_{\text {noise }}$ and $I_{\text {Back }}$, our results for the column densities would rise by just a factor of around 3. This is again due to the alleviating effect of the logarithm in Eq. (3), acting on the intensity contrast.

While for almost all of our clouds, peak column densities extracted from the GLIMPSE data are slightly higher than the values derived from the millimeter data, this difference does not correspond to the factor of 8 in resolution. Therefore, we compared the peak column densities extracted from different observational data for the previously studied IRDC 18223-3 (Beuther et al. 2002, 2005), located $3.7 \mathrm{kpc}$ from us with a $\mathrm{NH}_{3}$ rotation temperature of $\sim 33 \mathrm{~K}$ (Sridharan et al. 2005). For this particular cloud, $1.2 \mathrm{~mm}$ IRAM single-dish observations (Beuther et al. 2002), 8 micron Spitzer/GLIMPSE data, and interferometric data at 3.2 and $1.3 \mathrm{~mm}$ data obtained with the PdBI and SMA (Beuther et al. 2005, Fallscheer et al. 2009 in prep.) are available, the spatial resolution ranging between 11 arcsec and 1.4 arcsec (Table 2). For the GLIMPSE data, we obtained a columndensity distribution map according to the algorithm described in Sect. 3.2. Together with the $1.2 \mathrm{~mm}$ IRAM data shown as contours, this result is presented in Fig. 5. The peak column densities for all the millimeter data were calculated using Eq. (2), adopting the different beam sizes, and a temperature of $33 \mathrm{~K}$ (Sridharan et al. 2005) was always used. The peak flux density for the $3.2 \mathrm{~mm}$ PdBI data was taken from the corresponding paper (Beuther et al. 2005), while we (re-)assessed the peak flux densities for the IRAM ${ }^{2}$ and SMA data on the related FITS files, kindly provided by Beuther and Fallscheer. For the dust opacity per gram of dust, as for our SIMBA millimeter data, we always used the same opacity model, appropriate for coagulated dust particles with thin ice mantles (Ossenkopf \& Henning 1994, opacity column 5 in their Table 1). As we can see in Table 2, the peak column density derived from the high-resolution millimeter interferometry data is a factor of 40 higher than the one extracted with the mid-IR technique. A factor of a few between the mid-infrared and the millimeter single-dish results for the peak column densities can be accounted for by using other dust opacities/extinction cross sections or more extreme assumptions about the MIR foreground contribution (see above). However, the large difference between the mid-infrared and the millimeter interferometry results indicates a principle limitation of the extinction method in identifying high column density peaks. The realistically attainable intensity contrast at high optical depths determines this limit. In Table 2, we list the optical depths and image contrasts at 8 micron that would correspond to the column densities derived from the millimeter data. Obviously, realistic 8 micron images cannot provide such humongously high dynamic ranges required to derive column density levels similar to the interferometry results.

\section{Results}

\subsection{Morphology of the IRDCs}

The morphology of the clouds in our sample range between compact structures (IRDC 312.33-0.07 P1 and P2) and filaments (IRDC 309.13-0.15). They have sizes from 1' (IRDC 013.84$0.49 \mathrm{P} 2$ and P3) to $4^{\prime}$ (IRDC 317.71+0.11), which corresponds to roughly $1-3.5 \mathrm{pc}$ at the distance of these clouds. There is, in general, good agreement between the morphologies of the $1.2 \mathrm{~mm}$ emission and the $8 \mu \mathrm{m}$ extinction structures (see Figs. 3, 4). As a rule, dense areas of extinction material (IRDCs 013.84$0.49,313.72-0.29$ etc.) coincide with relatively bright compact sources at $1.2 \mathrm{~mm}$. For some IRDC complexes, some emission peaks at the millimeter wavelengths are associated with midIR emission sources (IRDCs 309.13-0.15 P2, 309.34-0.64 P2 and $317.71+0.11)$, which implies that they represent later evolutionary stages than those objects corresponding to $8 \mathrm{mi}-$ cron extinction. Among all clouds there is one particular case, IRDC 310.39-0.28, where the millimeter emission still peaks at the extinction maximum, despite the existence of a bright nearby MIR emission source ${ }^{3}$. The IRDCs 013.84-0.49, 312.33$0.07,318.13-0.34,320.23+0.32$, and $321.71+0.07$ exhibit several separated sources at mm wavelengths, coinciding with dense $8 \mu \mathrm{m}$ features. We note that IRDC 308.12-0.33 has an elongated shape and three recognizable substructures, one of them corresponding to the extinction maximum at mid-IR wavelengths. IRDC 309.13-0.15 shows two distinct $1.2 \mathrm{~mm}$ emission sources, a compact object (P1) coinciding with the MIR extinction region, and another (P2) that has an emission peak corresponding to the $8 \mu \mathrm{m}$ emission and an elongated tail. Extended millimeter emission, $2^{\prime} \times 4^{\prime}$ in size, with at least four substructures is present in IRDC 313.72-0.29. For IRDC 316.45-0.66, we can

\footnotetext{
2 The $1.2 \mathrm{~mm}$ peak flux density we find in the IRAM 30-m data is clearly higher than reported in Beuther et al. (2002). In that paper, the millimeter peaks had been fitted with Gaussians which occasionally underestimated the true peak flux densities.

3 Based on the similar LSR velocities for the MIR source and the extinction region, derived from our recent molecular line observations, both objects are probably associated.
} 

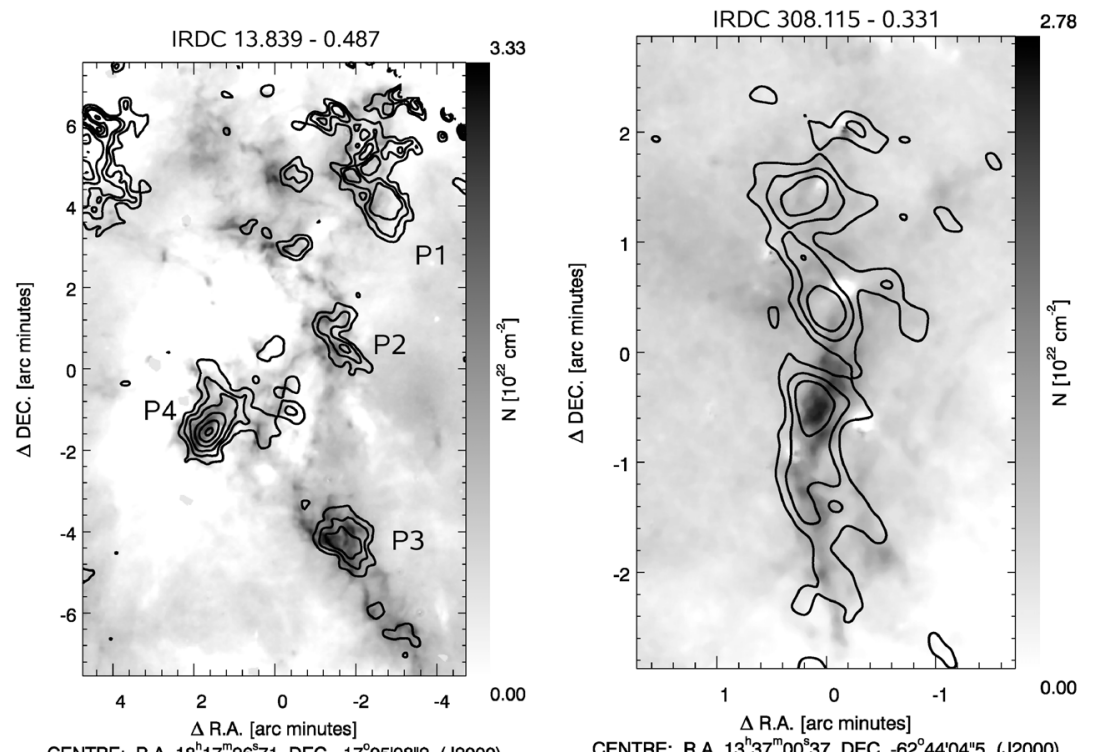

CENTRE: R.A. $18^{\mathrm{h}} 17^{\mathrm{m}} 26^{\mathrm{s}} .71$ DEC $-17^{\circ} 05^{\prime} 08^{\prime \prime 2}$ (J2000)

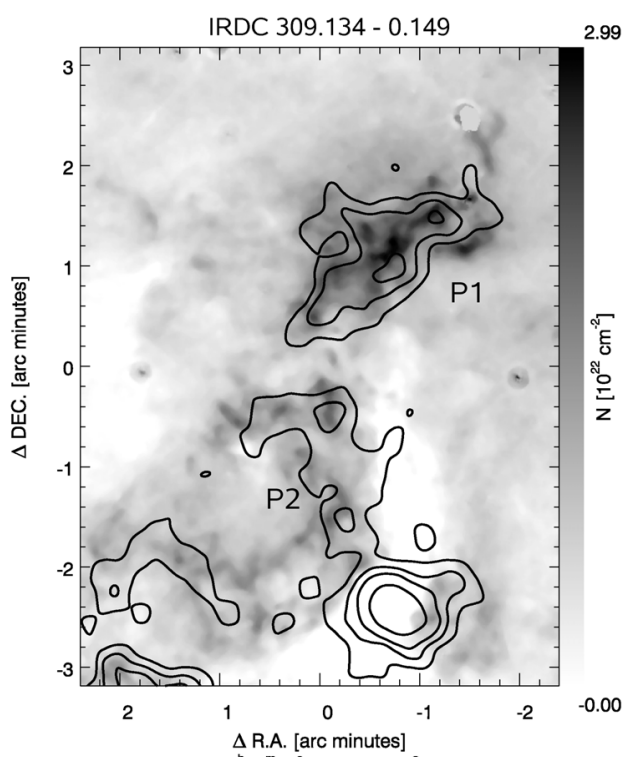

CENTRE: R.A. $13^{\mathrm{h}} 45^{\mathrm{m}} 23^{\mathrm{s}} .52$ DEC $-62^{\circ} 23^{\prime} 111^{\prime \prime 3}$ (J2000)
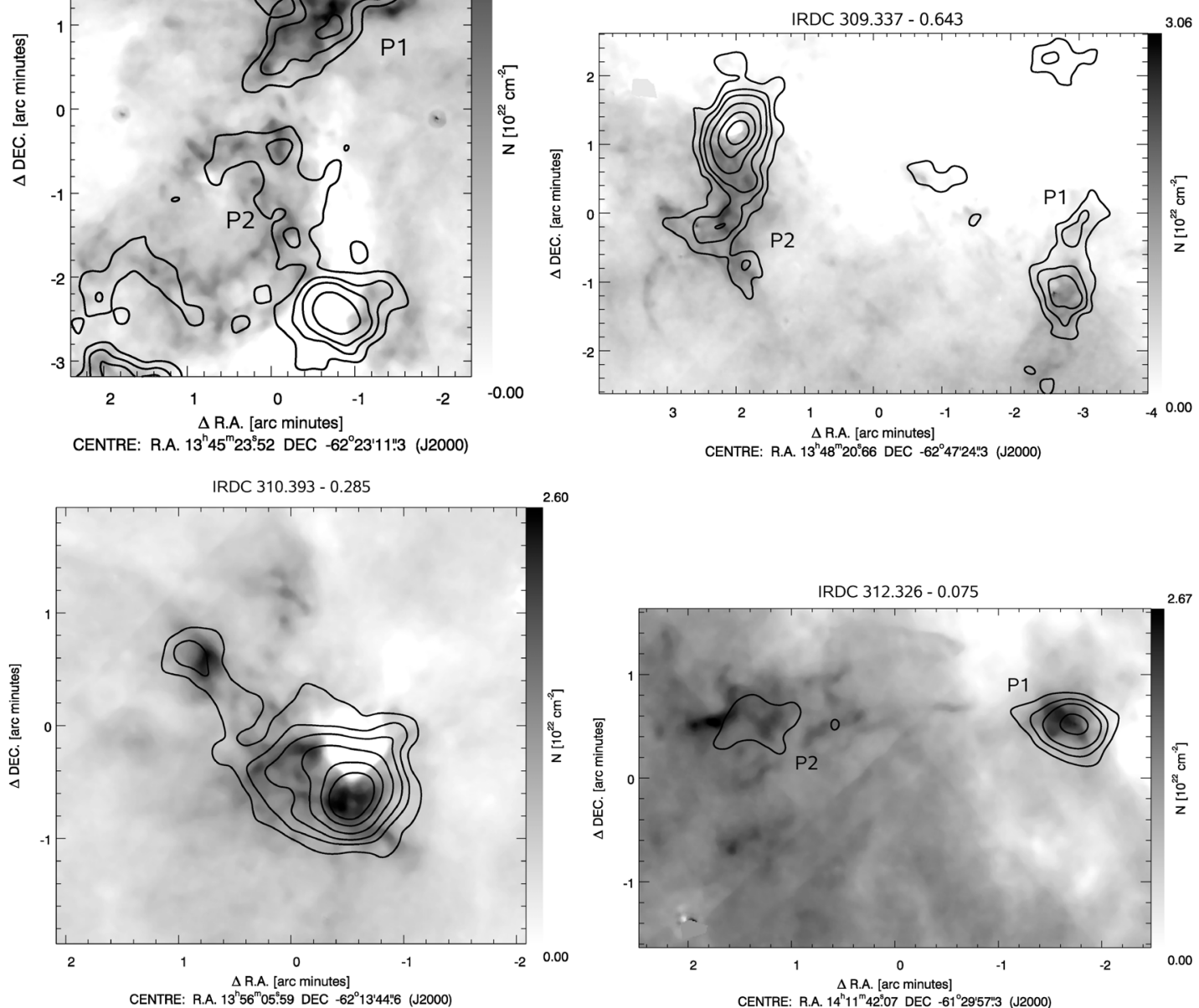

Fig. 3. Column density maps derived from $8 \mu \mathrm{m}$ extinction overlaid with $1.2 \mathrm{~mm}$ continuum emission as contours. The scaling is indicated in the bar to the right of each image. The contours are $60,108,156,240,360,480 \mathrm{mJy}^{\text {beam }}{ }^{-1}$ in all cases. 

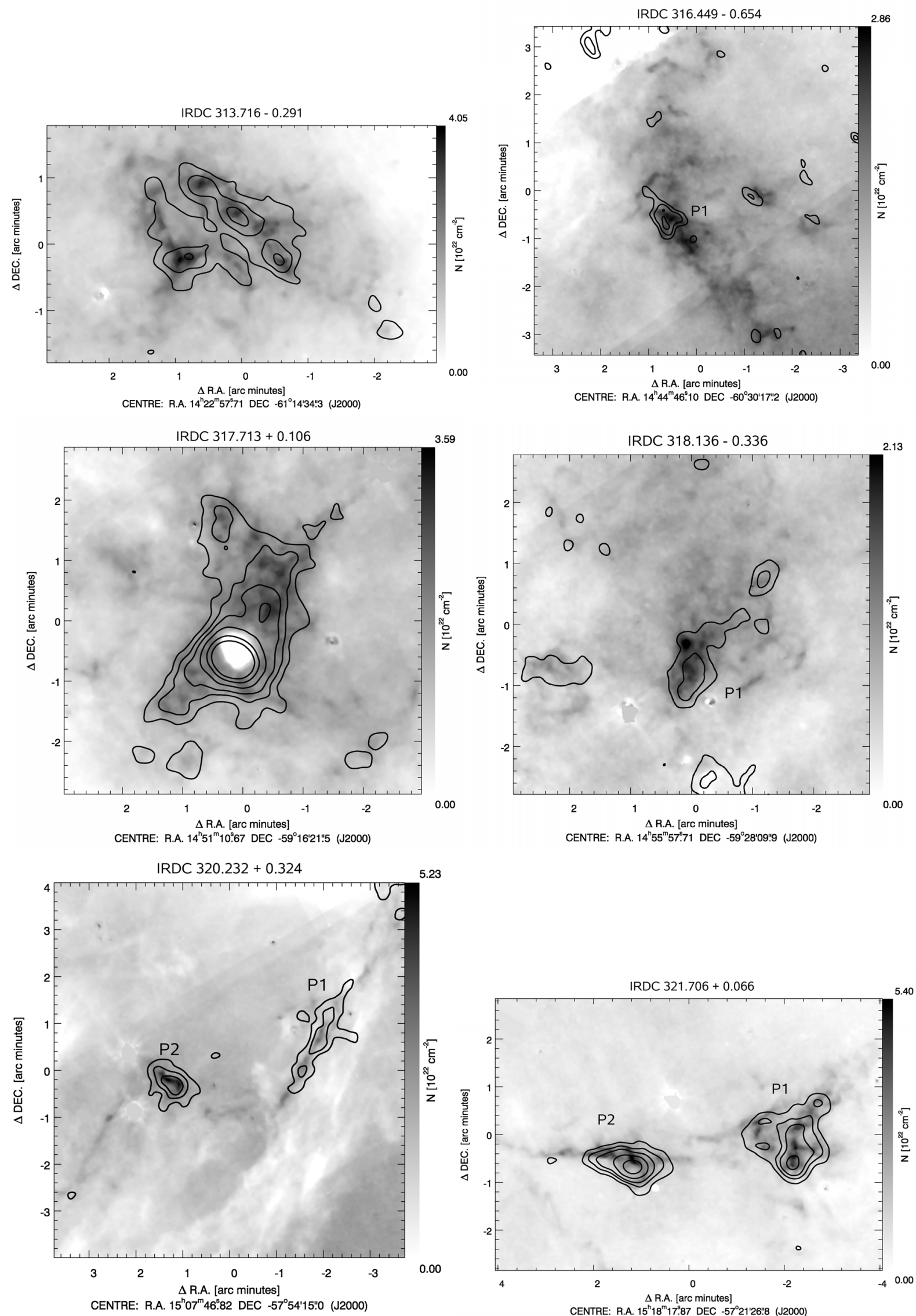

Fig. 4. Column density maps derived from $8 \mu \mathrm{m}$ extinction overlaid with $1.2 \mathrm{~mm}$ continuum emission as contours. The scaling is indicated in the bar to the right of each image. The contours are $60,108,156,240,360,480$ mJy beam $^{-1}$ in all cases except for IRDC $316.45-0.65$ which is 84 , $120,156,240,360,480$ mJy beam $^{-1}$ 


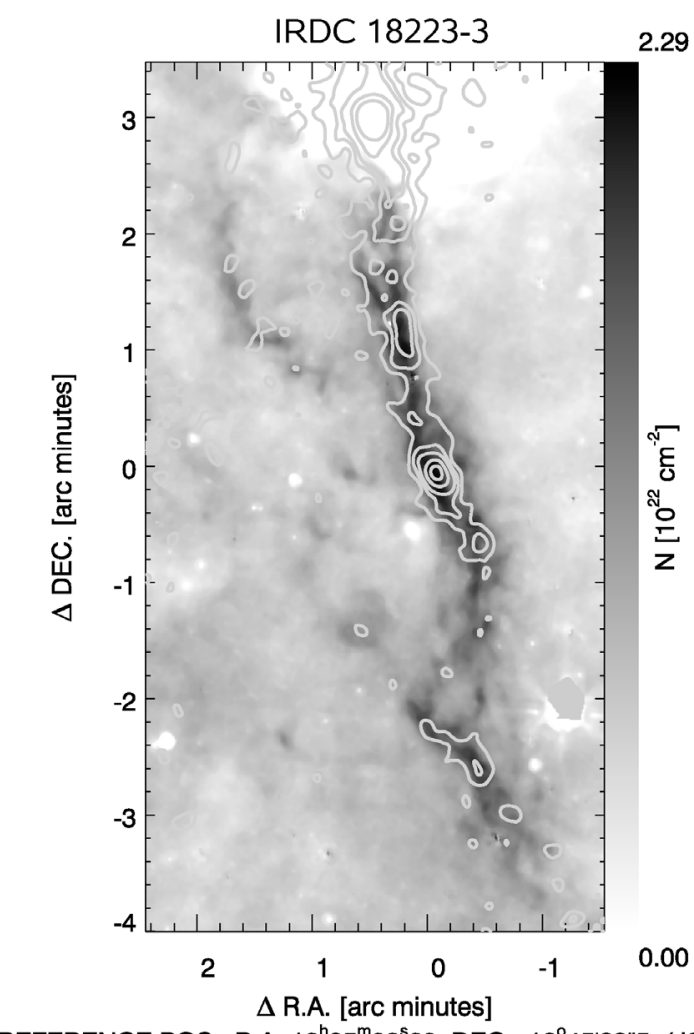

REFERENCE POS. R.A. $18^{\mathrm{h}} 25^{\mathrm{m}} 08^{\mathrm{s}} \cdot 60$ DEC $-12^{\circ} 45^{\prime} 23.5$ (J2000)

Fig. 5. Column density map derived from an $8 \mu \mathrm{m}$ Spitzer/GLIMPSE image overlaid with $1.2 \mathrm{~mm}$ IRAM continuum emission as contours. The image scaling is indicated in the sidebar. The contours are 38, 76, $114,190,266 \mathrm{mJy} \mathrm{beam}^{-1}$. The $(0,0)$ position corresponds to the center of the IRDC 18223-3.

recognise only one weak $1.2 \mathrm{~mm}$ source and diffuse, extended MIR extinction structures.

The positions of known IRAS sources are marked with plussigns in Figs. 1, 2, where the IRAC $8 \mu \mathrm{m}$ data are displayed as inverted greyscale images, and contours present the $1.2 \mathrm{~mm}$ data. In general, IRAS sources do not correspond to the extinction regions at $8 \mu \mathrm{m}$. In contrast, they agree with the locations where MIR emission coincides with millimeter emission peaks or just with the very bright MIR emission sources. In the case of IRDC $317.71+0.11$, the kinematic distance to one of the IRAS sources, located at the center of the $8 \mu \mathrm{m}$ emission, can be derived using the CS (2-1) line velocities reported by Bronfman et al. (1996) toward this IRAS source. The $v_{\text {LSR }}$ velocities of this $\mathrm{CS}$ measurement and our $\mathrm{HCO}^{+}$data for the neighbouring IRDC differ by less than $0.5 \mathrm{~km} \mathrm{~s}^{-1}$. The distance to the cloud and the IRAS source is then around $2.9 \mathrm{kpc}$. Hence, assuming that the dark cloud is related to this IRAS point source, the infrared luminosity of the compound (IR source + IRDC) is $\approx 10^{4} L_{\odot}$, using the IRAS approximation formula from Henning et al. (1990), which is the typical luminosity of pre-main-sequence OB stars.

\subsection{Masses and column densities}

Millimeter continuum emission was detected toward all 12 clouds and column density maps were extracted from $8 \mu \mathrm{m}$ images by using the algorithm described in Sect. 3.2. Figures 3, 4 present the column density maps of every region, superimposed on the corresponding $1.2 \mathrm{~mm}$ contours. In Table 3, the properties of the IRDCs are compiled: name, right ascension, declination, distance, peak flux density, integrated flux density, mass and peak column density of the $1.2 \mathrm{~mm}$ sources, and mass and peak column density of the extinction matter. For every cloud in the table, the first line corresponds to the total millimeter flux density as well as the total masses of the emission and extinction matter. The following lines present data for the separate millimeter subclumps, which are labeled with $\mathrm{P}$.

Where appropriate, we distinguished between the separate subclumps at $1.2 \mathrm{~mm}$. In Figs. 3, 4 and Table 3, these separate emission sources are named with the designation "P" (e.g. P1, P2). For all of them, we measured peak flux density and integrated flux density, derived masses, and column densities according to Eq. (1)-(2). In the case of IRDCs 309.13-0.15 (P2), 309.34-0.64 (P2), and 317.71+0.11 (P1) the parts coinciding with strong $8 \mu \mathrm{m}$ emission features were not taken into account when measuring $1.2 \mathrm{~mm}$ flux densities and hence for estimating masses and column densities. The typical range of masses of the separate millimeter sources with $T=20 \mathrm{~K}$ was $50-1000 M_{\odot}$, and the column density range between 0.9 and $4.6 \times 10^{22} \mathrm{~cm}^{-2}$.

In calculating the total masses of the extinction material in individual clumps, we considered regions above 3 sigma, where sigma is in this case the standard deviation in the full extinction maps. The total masses of the IRDCs for extinction matter were found to range from 300 to $1700 M_{\odot}$ and the derived peak column densities correspond to values from 2.1 to $5.4 \times 10^{22} \mathrm{~cm}^{-2}$.

\subsection{Comparison with results for other cores}

We compare our results with previously obtained characteristics for low- and high-mass pre-stellar cores.

\subsubsection{Comparison with low-mass cores}

Extinction mapping has been applied mainly along the lines of the near-infrared colour excess method (e.g., Lombardi \& Alves 2001; Lombardi 2008) or classical star-count-techniques in the visible or near-infrared (e.g., Dobashi et al. 2005; Froebrich et al. 2005). These approaches are most powerful towards mediumextinction regions. In contrast, the extinction-map method used in the current paper exploits the extinction of mid-infrared extended emission and hence does not rely on the identification of stellar background sources. This method can peak into cores of column densities as high as $10^{23} \mathrm{~cm}^{-2}$. It was used in the analysis of low-mass starless cores based on ISOCAM data (Bacmann et al. 2000), which provides an opportunity to compare our results. To compare fairly, two effects must be considered: (a) Bacmann et al. (2000) used the standard Draine \& Lee (1984) extinction cross sections. These are more than a factor of 3 smaller than the Weingartner \& Draine (2001) values we use. We therefore recomputed the peak column densities reported in Bacmann et al. (2000) for the low-mass cores by adopting the Weingartner \& Draine (2001) dust-extinction model; and (b) for the low-mass cores, which typically reside at distances of less than $300 \mathrm{pc}$, the linear spatial resolution is much better than in the case of IRDCs with their distances of 2-5 kpc. Therefore, the true column density peaks are more sharply resolved in the low-mass case. To assess the effect of poor spatial resolution on our derived peak column densities, we used a synthetic column density map derived in Steinacker et al. (2005) for one of the low-mass cores of Bacmann et al. (2000), namely Rho Oph D $(d=160 \mathrm{pc})$. We convolved this map with kernels appropriate for emulating the much coarser linear resolution toward our 
Table 3. Properties of the IRDCs. For every cloud the first line corresponds to the total mass, the following to the masses of separate millimeter sources.

\begin{tabular}{|c|c|c|c|c|c|c|c|c|c|c|}
\hline Name & $\begin{array}{c}\text { RA } \\
(\mathrm{J} 2000.0)\end{array}$ & $\begin{array}{c}\text { Dec. } \\
(\mathrm{J} 2000.0)\end{array}$ & $\begin{array}{c}D \\
(\mathrm{kpc})\end{array}$ & $\begin{array}{l}\text { Peak Flux density } \\
\text { (mJy) }\end{array}$ & $\begin{array}{l}\text { Integrated Flux density } \\
\qquad(\mathrm{Jy})\end{array}$ & $\begin{array}{l}\text { Mass } 1.2 \mathrm{~mm} \\
\left(M_{\odot}\right)\end{array}$ & $\begin{array}{l}N(1.2 \mathrm{~mm})^{1} \\
\left(10^{22} \mathrm{~cm}^{-2}\right)\end{array}$ & $\begin{array}{c}N_{0}(1.2 \mathrm{~mm})^{2} \\
\left(10^{22} \mathrm{~cm}^{-2}\right)\end{array}$ & $\begin{array}{c}\text { Mass }(8 \mu \mathrm{m}) \\
\left(M_{\odot}\right)\end{array}$ & $\begin{array}{c}N(8 \mu \mathrm{m}) \\
\left(10^{22} \mathrm{~cm}^{-2}\right)\end{array}$ \\
\hline IRDC 308.12-0.33 & 133701.2 & -624440 & 4.32 & 225 & 1.88 & 580 & 1.7 & 60.3 & $520_{-30}^{+40}$ & $2.8_{-0.16}^{+0.16}$ \\
\hline IRDC 309.13-0.15 & \multirow[t]{3}{*}{134517.1} & \multirow[t]{3}{*}{-622157} & \multirow[t]{3}{*}{3.92} & $330^{3}$ & $4.39^{3}$ & $1150^{3}$ & $2.6^{3}$ & \multirow{3}{*}{41.9} & $1750_{-310}^{+370}$ & \multirow[t]{3}{*}{$3.0_{-0.13}^{+0.13}$} \\
\hline $\mathrm{P} 1$ & & & & 162 & 1.37 & 360 & 1.3 & & & \\
\hline $\mathrm{P} 2$ & & & & 124 & 0.96 & 250 & 0.9 & & & \\
\hline IRDC 309.34-0.64 & \multirow[t]{3}{*}{134839.8} & \multirow[t]{3}{*}{-624726} & \multirow[t]{3}{*}{3.46} & $504^{3}$ & $3.69^{3}$ & $750^{3}$ & $3.9^{3}$ & \multirow{3}{*}{48.5} & $750_{-150}^{+170}$ & \multirow[t]{3}{*}{$3.1_{-0.24}^{+0.24}$} \\
\hline $\mathrm{P} 1$ & & & & 216 & 0.94 & 190 & 1.7 & & & \\
\hline $\mathrm{P} 2$ & & & & 155 & 0.81 & 170 & 1.3 & & & \\
\hline IRDC $310.39-0.28$ & 135601.7 & -621427 & 4.93 & 594 & 2.48 & 1029 & 4.6 & 186.1 & $1320_{-50}^{+60}$ & $2.6_{-0.16}^{+0.16}$ \\
\hline IRDC $312.33-0.07$ & \multirow[t]{3}{*}{141156.8} & \multirow[t]{3}{*}{-612925} & \multirow[t]{3}{*}{4.05} & 288 & 0.77 & 210 & 2.3 & \multirow[t]{3}{*}{76.6} & \multirow[t]{3}{*}{$290_{-60}^{+100}$} & \multirow[t]{3}{*}{$2.7_{-0.16}^{+0.33}$} \\
\hline $\mathrm{P} 1$ & & & & 288 & 0.46 & 130 & 2.3 & & & \\
\hline $\mathrm{P} 2$ & & & & 92 & 0.31 & 90 & 0.7 & & & \\
\hline IRDC $313.72-0.29$ & 142305.4 & -611448 & 3.33 & 172 & 1.98 & 370 & 1.3 & 35.7 & $700_{-70}^{+90}$ & $4.1_{-0.16}^{+0.16}$ \\
\hline IRDC $316.45-0.66$ & \multirow[t]{2}{*}{144450.4} & \multirow[t]{2}{*}{-603054} & \multirow[t]{2}{*}{3.01} & 159 & 0.96 & 150 & 1.3 & \multirow[t]{2}{*}{32.3} & \multirow{2}{*}{$430_{-70}^{+80}$} & \multirow{2}{*}{$2.9_{-0.16}^{+0.16}$} \\
\hline $\mathrm{P} 1$ & & & & 159 & 0.58 & 90 & 1.3 & & & \\
\hline IRDC $317.71+0.11$ & \multirow[t]{2}{*}{145107.5} & \multirow[t]{2}{*}{-591611} & \multirow[t]{2}{*}{2.9} & $954^{3}$ & $8.00^{3}$ & $1150^{3}$ & $7.5^{3}$ & & \multirow[t]{2}{*}{$1320_{-150}^{+150}$} & \multirow[t]{2}{*}{$3.6_{-0.15}^{+0.15}$} \\
\hline $\mathrm{P} 1$ & & & & 250 & 1.98 & 280 & 2.0 & 47.9 & & \\
\hline IRDC $318.13-0.34$ & \multirow[t]{2}{*}{145558.4} & \multirow[t]{2}{*}{-592831} & 2.96 & 142 & 2.48 & 370 & 1.1 & 26.9 & $680_{-140}^{+160}$ & $2.1_{-0.16}^{+0.16}$ \\
\hline $\mathrm{P} 1$ & & & & 142 & 1.02 & 150 & 1.1 & & & \\
\hline IRDC $320.23+0.32$ & 150756.7 & -575427 & 1.97 & 186 & 1.68 & 110 & 1.5 & 24.6 & $600_{-250}^{+320}$ & $5.2_{-0.16}^{+0.16}$ \\
\hline $\mathrm{P} 1$ & & & & 142 & 1.00 & 70 & 1.1 & & & \\
\hline $\mathrm{P} 2$ & & & & 186 & 0.68 & 50 & 1.5 & & & \\
\hline IRDC $321.71+0.07$ & 151826.7 & -572156 & 2.14 & 408 & 3.12 & 240 & 3.2 & 56.9 & $460_{-80}^{+80}$ & $5.4_{-0.23}^{+0.16}$ \\
\hline $\mathrm{P} 1$ & & & & 276 & 1.69 & 130 & 2.2 & & & \\
\hline $\mathrm{P} 2$ & & & & 408 & 1.43 & 110 & 3.2 & & & \\
\hline IRDC 013.84-0.49 & 181721.2 & -170923 & 2.66 & 438 & 9.38 & 1130 & 3.5 & 77.0 & $1150_{-120}^{+130}$ & $3.3_{-0.13}^{+0.13}$ \\
\hline $\mathrm{P} 1$ & & & & 214 & 2.97 & 360 & 1.6 & & & \\
\hline $\mathrm{P} 2$ & & & & 163 & 1.12 & 130 & 1.3 & & & \\
\hline P3 & & & & 192 & 1.45 & 170 & 1.5 & & & \\
\hline P4 & & & & 438 & 3.54 & 430 & 3.5 & & & \\
\hline
\end{tabular}

1 Peak column density per beam.

${ }^{2}$ Extrapolated column density, obtained by applying the correction factor explained in Sect. 4.3.2

3 This mm peak corresponds to a mid-infrared bright source near to the IRDC.

IRDC targets and computed the ratio of the unsmoothed to the smoothed peak column density value. These factors (typically in the range from 1.5 to 3.5 ) were multiplied by the peak column densities we have originally derived from the IRDC extinction maps.

A comparison between the column densities for low-mass cores and our IRDCs, after taking into account the afore mentioned considerations (a) and (b), is shown in Fig. 6. We can see a clear trend of high-mass cores having higher column densities than low-mass objects. This qualitative difference shows that IRDCs are not just distant Taurus-like clouds, but a distinct type of clouds with the potential of forming a distinct type of stars (see below and Sect. 4.4).

In step (b), we note that we applied a correction factor that increases the column densities with increasing kinematic distance. To fortify statistically our statement that Infrared Dark Clouds have "stochastically larger" values of column densities than low-mass pre-stellar cores, we used the (Wilcoxon)-Mann-Whitney U one-tailed test (e.g., Wall \& Jenkins 2003). It is a non-parametric test for assessing whether two samples of observations originate in the same distribution or not. It operates well for a small number of observations in one sample. The null hypothesis is that the two samples are drawn from a single population. For the test, we used low-mass column density values from Bacmann et al. (2000) adapted to the Weingartner \& Draine (2001) dust extinction model and column densities for IRDCs without applying the linear spatialresolution correction (b) mentioned above.

For both populations, we computed the Rank Sum within the nonparametric Mann-Whitney statistic, usually called U (108 versus 0 ). The distribution of the U-statistic for the null hypothesis is known and can be found in special tables (e.g., Siegel $\&$ Castellan 1988). We then estimated the probability that the values for low-mass objects and IRDCs originate in the same distribution. The derived probability of lower than 0.005 rejects the null hypothesis and demonstrates that our two samples come from different distributions.

\subsubsection{Comparison with high-mass cores}

The high-mass clumps that we want to compare have all been observed at $1.2 \mathrm{~mm}$, and comprise the following samples: highmass starless-core candidates (HMSCs) (Sridharan et al. 2005; Beuther et al. 2002), Infrared Dark Clouds from Rathborne et al. (2006), and results presented in this paper for our $1.2 \mathrm{~mm}$ data. To (re-)calculate peak column densities for the first two sets of data, we also used Eq. (2). In the case of HMSCs, peak flux densities were taken from Beuther et al. (2002), and distance and temperature estimates for all these objects come from Sridharan et al. (2005). In Rathborne et al. (2006), peak flux densities and distances for all 38 IRDCs are presented. For the calculation, 


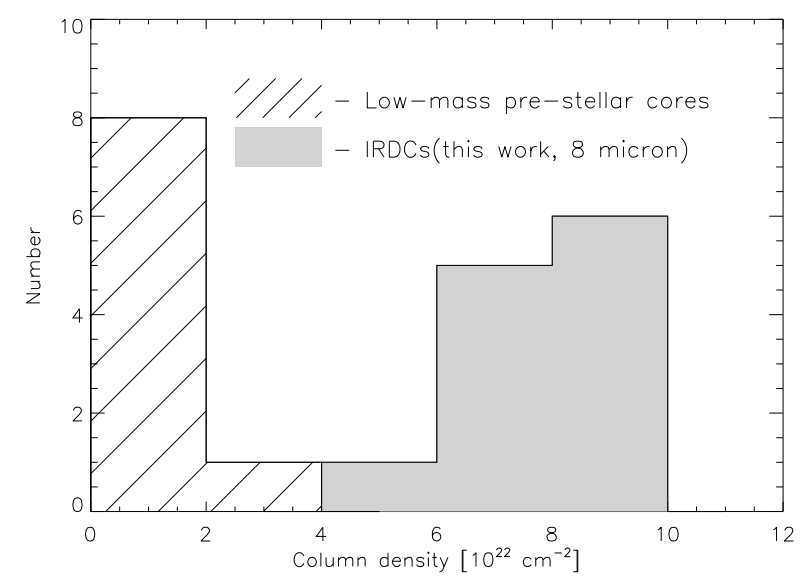

Fig. 6. Histogram showing the principle distribution of column densities for a collection of low-mass cores from Bacmann et al. (2000) (after taking into account consideration (a) from Sect. 4.3.1) and for the IRDCs presented here (after taking into account consideration (b) from Sect. 4.3.1). The Mann-Whitney-U one-tailed test confirms the clear separation of the two distributions even when the different spatial resolutions (consideration (b)) are not taken into account (Sect. 4.3.1).

we assume again $20 \mathrm{~K}$ for the temperature, and as dust opacity $\varkappa_{v}$ and gas-to-dust mass ratio we adopt the values $1.0 \mathrm{~cm}^{2} \mathrm{~g}^{-1}$ and 100 , respectively. Hence, we use the same parameters as for the analysis of our $1.2 \mathrm{~mm}$ data, except for the different beam size, which equals $\left(11^{\prime \prime}\right)^{2}$ in steradians, adapted to the IRAM 30-m telescope.

The measured data remain affected by the convolution with the observational beam, which results in different linear smoothing scales for objects at different distances. To eliminate this smoothing effect, we attempted to extrapolate the measured peak column densities per beam back to their true values, assuming an (analytic) column density profile. But what is an appropriate choice for such a power law? A first idea is to use the data at different beam sizes that we have for IRDC 18223-3 (Sect. 3.3). However, it turns out that these data sets provide no unique trend for such a power law. The comparison between the IRAM 30-m data and the SMA data imply a relatively steep power law of $N \sim r^{-1.75}$, and the comparison with the PdBI data would even indicate a much steeper power law index. The fact that these two data sets do not result in a similar power law may be related to the different degrees to which these observations are able to recover extended emission. Furthermore, at the high spatial resolution of the SMA observations, we begin to see a dense rotation structure (Fallscheer et al. 2009, in prep.) that is distinct from the remainder of the surrounding clump. However, we also compared the column density values of a few other IRDCs, observed both at single-dish and interferometric resolution (Rathborne et al. 2006, 2008). Again, no clear trend for a certain power law range is obvious. According to Johnstone et al. (2003), the column density profile of the filament structure of the famous IRDC G11.11-0.02 should be very steep and may reach $\sim r^{-3}$. Such a significant difference to commonly observed values might be an additional feature characterizing massive star-forming clumps, especially if filamentary structures are involved. On the other hand, Bacmann et al. (2000) showed that $N \sim r^{-1}$ can be a reasonable choice for lower-mass starless cores, and also the single dish mapping of young massive clumps by Beuther et al. (2002) resulted in less steep power laws for the column density quite close to the low-mass core results. Hence,

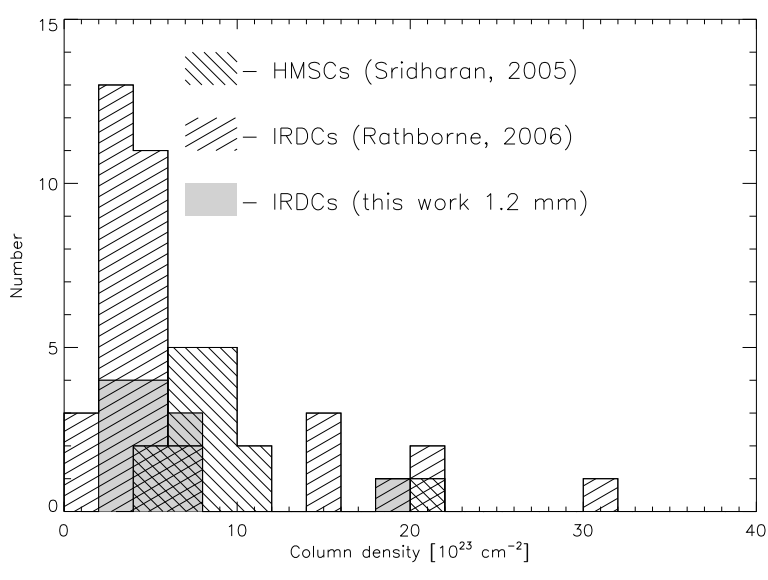

Fig. 7. Distribution of extrapolated column densities for the HMSCs from Sridharan et al. (2005), the IRDCs from Rathborne et al. (2006), and the IRDCs from the present paper. Note that we have applied the corrections and extrapolations mentioned in Sect. 4.3.2 for all these high-mass cores.

by assuming $N \sim r^{-1}$ in extrapolating the true column densities, we can obtain a robust lower limit.

To recalculate all column density values, an artificial column density distribution map was created (by just assuming an analytical $N \sim r^{-1}$ profile) with a peak in the centre of the array normalised to 1.0 and the assumption that 1 pixel corresponds to $2000 \mathrm{AU}$ (a typical length scale for fragmentation). Then, for every source from the three high-mass samples mentioned above, this column density distribution map was convolved with a smoothing Gaussian kernel emulating the effect of observing at mm wavelengths with single-dish telescopes (11-24" beam). The kernel size (FWHM) is computed by taking the ratio of the effective linear resolution (in AU) achieved within the observed mm maps to the $2000 \mathrm{AU}$ pixel size from the artificial map. After convolving the artificial map with such a Gaussian kernel, the peak column densities in these synthetic maps become smaller than 1 . The correction factor for every source is then the ratio of the original peak column density value (1.0) to the peak column density value after beam convolution. These factors are then applied to the column density values derived according to Eq. (2), which then approximates the true peak column density values. These extrapolated values for the new regions reported here are also given in Col. 9 of Table 3.

Figure 7 summarizes the distribution of the extrapolated peak column densities that we finally obtained. The distribution of the true peak column densities indicates a similar order of magnitude for previously obtained values for the two other samples of massive star-forming regions and our new SIMBA results. Most of the clouds have (extrapolated) column densities in the range from 1.0 to $12.0 \times 10^{23} \mathrm{~cm}^{-2}$ with few exceptions reaching $20.0-30.0 \times 10^{23} \mathrm{~cm}^{-2}$.

\subsection{Comparison with theoretical models}

Infrared Dark Clouds, located several kpc away from us, have high masses, volume and column densities, but "high" values alone do not guarantee that they are really the progenitors of massive stars, and we need additional criteria to estimate this possibility. According to Krumholz \& McKee (2008), only clouds with column densities of at least $1 \mathrm{~g} \mathrm{~cm}^{-2}$, which corresponds to $3 \times 10^{23} \mathrm{~cm}^{-2}$ in our units, can form massive stars. 
As we can see in Table 3, the direct transformation of observational results for IRDCs from our list indicates peak column densities one order of magnitude lower than this limit. On the other hand, it was shown in Sect. 4.3 that the directly-observed peak column densities are still affected by the convolution with the beam of the millimeter observations. After taking this into account, the derived values of the extrapolated column density increase by a factor of 10 or higher (see Fig. 7). We can thus, reach the $3 \times 10^{23} \mathrm{~cm}^{-2}$ threshold in the case of the infrared dark clouds.

Since we observe not only compact sources, but also very filamentary structures such as IRDC $320.23+0.32 \mathrm{P} 1$, this raises the question, of whether it is possible to form massive stars in these structures. Banerjee \& Pudritz (2008) demonstrated that filaments play a dominant role in controlling the physics, accretion rate, and angular momentum of the far smaller-scale accretion disk that forms within these collapsing structures. Large-scale filamentary flows sustain a high accretion rate $(\dot{M} \sim$ $\left.10^{-2} M_{\odot} \mathrm{yr}^{-1}\right)$ due to the supersonic gas flow onto the protostellar disk. These rates are $10^{3}$ times higher than predicted by the collapse of the singular isothermal spheres and exceed the accretion rates necessary to squeeze the radiation field of the newlyborn massive star. Thus, for almost all our clouds, we confirm the possibility of forming massive stars.

\section{Conclusions}

We have discussed our progress in understanding the properties of Infrared Dark Clouds. A set of 12 clouds located in the southern hemisphere has been selected from the MSX 8.3 micron images. For these clouds, $1.2 \mathrm{~mm}$ maps were obtained with the SIMBA bolometer array at the SEST telescope. GLIMPSE midinfrared images of these regions were retrieved from the Spitzer Archive.

The new sources exhibit a variety of IRDC morphologies, from compact cores to filamentary shaped ones, from infrared quiet examples (no Spitzer $8 \mu \mathrm{m}$ emission sources) to more active ones. As a rule, our sample shows good agreement between the morphologies of $1.2 \mathrm{~mm}$ emission and $8 \mu \mathrm{m}$ extinction features. The total masses of the IRDCs were found to range from 150 to $1150 M_{\odot}$ (emission data) and from 300 to $1750 M_{\odot}$ (extinction data). We derived peak column densities of between 0.9 and $4.6 \times 10^{22} \mathrm{~cm}^{-2}$ (emission data) and 2.1 and $5.4 \times 10^{22} \mathrm{~cm}^{-2}$ (extinction data).

Since the MIR extinction method was used previously for the analysis of low-mass starless cores, we check how our findings relate to the published results. To complete a fair comparison, we used the same dust model and the same spatial resolution in both cases. It is shown, that there is a clear trend for the highmass cores to attain higher column densities than the low-mass objects. This qualitative difference means that most IRDCs are not just distant Taurus-like clouds, but a distinct type of clouds with the potential to form a distinct type of stars. A simple statistical analysis (the Mann-Whitney-U one-tailed test) confirms this statement also when the different spatial resolutions are not taken into account and thus the IRDC column densities are underestimated.

Using the data from two different regions of the spectrum and applying two different techniques for estimating IRDC parameters enables us to compare these two methods. On the one hand, the extinction technique has some advantages over the millimeter technique. It is a cheap method since GLIMPSE at $8 \mu \mathrm{m}$ has covered large parts of the Galactic plane in the 4 th and 1st quadrant. The GLIMPSE data have a higher spatial resolution than millimeter single-dish data, which reveals the often filigree substructures of the clouds. Furthermore, this method does not depend on assumptions about the temperature of the IRDCs in estimating masses and column densities. However, our comparison shows that the extinction method has a principle limitation in distinguishing very high image contrasts and hence of identifying high column density peaks $\gg 10^{23} \mathrm{~cm}^{-2}$. Hence, with the extinction method, we can estimate only a lower limit to the column density values, despite the high resolution. The limit is around $A_{\mathrm{V}}=75 \mathrm{mag}$ when applying the Weingartner \& Draine (2001) $R_{\mathrm{V}}=5.5 \mathrm{~B}$ extinction law (corresponding to roughly 200 mag when following the common $R_{\mathrm{V}}=3.1 \mathrm{ex}-$ tinction law reviewed in Mathis 1990). High-spatial resolution (sub-)millimeter observations are hence crucial in assessing even higher column density ranges and to identifying the true column density maxima.

To compare column densities extracted with the emission method with previously obtained values for IRDCs and HMPOs, we extrapolated them back to the true peak column densities by assuming a column density profile of $\sim r^{-1}$, and thus, mitigating the spatial resolution differences within the different samples. The distribution of the true peak column densities indicates a similar order of magnitude for our new SIMBA results and the two other samples of massive star-forming regions. Moreover, the true peak column densities exceed the theoretical limit of $3 \times 10^{23} \mathrm{~cm}^{-2}$ (or $1 \mathrm{~g} \mathrm{~cm}^{-2}$ ), which has been proposed as a means of identifying potentially high-mass star-forming clouds.

Thus, extracted values for masses and column densities both for emission and extinction matter show a clear difference between IRDCs and known low-mass pre-stellar cores, and confirm our assumptions that Infrared Dark Clouds can present the earliest stages of high-mass star formation.

Acknowledgements. We thank Jürgen Steinacker for discussions and for providing us with the synthetic column density map of Rho Oph D. We are indebted to Henrik Beuther and Cassandra Fallscheer for discussions and for making available the data for IRDC 18223-3 to us in electronic form. We wish to thank Maxim Voronkov for help with observations with the Australian MOPRA telescope. This research has made use of the NASA/ IPAC Infrared Science Archive, which is operated by the Jet Propulsion Laboratory, California Institute of Technology, under contract with the National Aeronautics and Space Administration. NASA's Astrophysics Data System was used to assess the literature given in the references.

\section{References}

Bacmann, A., André, P., Puget, J.-L., et al. 2000, A\&A, 361, 555 Banerjee, R., \& Pudritz, R. E. 2008, ed. H. Beuther, H. Linz, \& T. Henning, ASP Conf. Ser., 387, 216

Benjamin, R. A., Churchwell, E., Babler, B. L., et al. 2003, PASP, 115, 953 Beuther, H., Schilke, P., Menten, K. M., et al. 2002, ApJ, 566, 945 Beuther, H., Sridharan, T. K., \& Saito, M. 2005, ApJ, 634, L185

Beuther, H., Churchwell, E. B., McKee, C. F., \& Tan, J. C. 2007, in Protostars and Planets V, ed. B. Reipurth, D. Jewitt, \& K. Keil, 165

Bronfman, L., Nyman, L.-A., \& May, J. 1996, A\&AS, 115, 81

Carey, S. J., Clark, F. O., Egan, M. P., et al. 1998, ApJ, 508, 721

Carey, S. J., Feldman, P. A., Redman, R. O., et al. 2000, ApJ, 543, L157

Diolaiti, E., Bendinelli, O., Bonaccini, D., et al. 2000, in Adaptive Optical Systems Technology, Presented at the Society of Photo-Optical Instrumentation Engineers (SPIE) Conference, ed. P. L. Wizinowich, Proc. SPIE, 4007, 879

Dobashi, K., Uehara, H., Kandori, R., et al. 2005, PASJ, 57, 1

Draine, B. T., \& Lee, H. M. 1984, ApJ, 285, 89

Egan, M. P., Shipman, R. F., Price, S. D., et al. 1998, ApJ, 494, L199

Froebrich, D., Ray, T. P., Murphy, G. C., \& Scholz, A. 2005, A\&A, 432, L67

Henning, T., Pfau, W., \& Altenhoff, W. J. 1990, A\&A, 227, 542

Indebetouw, R., Mathis, J. S., Babler, B. L., et al. 2005, ApJ, 619, 931

Johnstone, D., Fiege, J. D., Redman, R. O., Feldman, P. A., \& Carey, S. J. 2003, ApJ, 588, L37 
Krumholz, M. R. \& McKee, C. F. 2008, Nature, 451, 1082 Levine, E. S., Heiles, C., \& Blitz, L. 2008, ApJ, 679, 1288 Lombardi, M. 2008, ArXiv e-prints

Lombardi, M., \& Alves, J. 2001, A\&A, 377, 1023

Mathis, J. S. 1990, ARA\&A, 28, 37

Nyman, L.-Å., Lerner, M., Nielbock, M., et al. 2001, The Messenger, 106, 40 Ossenkopf, V., \& Henning, T. 1994, A\&A, 291, 943

Perault, M., Omont, A., Simon, G., et al. 1996, A\&A, 315, L165

Peretto, N., Fuller, G. A., André, P., \& Hennebelle, P. 2008, ed. H. Beuther, H. Linz, \& T. Henning, 50

Pillai, T., Wyrowski, F., Carey, S. J., \& Menten, K. M. 2006a, A\&A, 450, 569

Pillai, T., Wyrowski, F., Menten, K. M., \& Krügel, E. 2006b, A\&A, 447, 929

Rathborne, J. M., Jackson, J. M., \& Simon, R. 2006, ApJ, 641, 389

Rathborne, J. M., Jackson, J. M., Zhang, Q., \& Simon, R. 2008, ArXiv e-prints, 808

Saito, H., Mizuno, N., Moriguchi, Y., et al. 2001, PASJ, 53, 1037
Siegel, S., \& Castellan, N. J. 1988, Nonparametric Statistics for the Behavioural Sciences (McGraw-Hill)

Simon, R., Jackson, J. M., Rathborne, J. M., \& Chambers, E. T. 2006a, ApJ, 639, 227

Simon, R., Rathborne, J. M., Shah, R. Y., Jackson, J. M., \& Chambers, E. T. 2006b, ApJ, 653, 1325

Sridharan, T. K., Beuther, H., Saito, M., Wyrowski, F., \& Schilke, P. 2005, ApJ, 634, L57

Steinacker, J., Bacmann, A., Henning, T., Klessen, R., \& Stickel, M. 2005, A\&A, 434,167

Wall, J. V., \& Jenkins, C. R. 2003, Practical Statistics for Astronomers (Princeton Series in Astrophysics)

Weferling, B., Reichertz, L. A., Schmid-Burgk, J., \& Kreysa, E. 2002, A\&A, 383,1088

Weingartner, J. C., \& Draine, B. T. 2001, ApJ, 548, 296

Zinnecker, H., \& Yorke, H. W. 2007, ARA\&A, 45, 481 